presented for care, the majority were able to return to work. A major medical challenge encountered was responding to a serious GI illness outbreak. Future medical planning will focus on provision of pharmacy services and promoting the use of eye personal protective equipment in wildfire hazard zones.

Prehosp Disaster Med 2017;32(Suppl. 1):s124-s125

doi:10.1017/S1049023X17003521

Discovering Best Practice for the Implementation of Evacuation Centers for Vulnerable Populations: Findings from a Japanese Pilot Study Mayumi Kako ${ }^{1}$, Yosuke Takada ${ }^{2}$, Paul Arbon ${ }^{3}$, Malinda Steenkamp ${ }^{4}$, Benjamin J. Ryan ${ }^{5}$

1. School Of Nursing and Midwifery, Flinders University, Torrens Resilience Institute, Adelaide/SA/Australia

2. Disaster Reduction and Human Renovation Institution, Kobe/ Japan

3. Torrens Resilience Institution, Adelaide/SA/Australia

4. Torrens Resilience Institute, Adelaide/ACT/Australia

5. College Of Public Health, Medical and Veterinary Sciences, James Cook University, Townsville/QLD/Australia

Study/Objective: This paper will report the preliminary findings of a pilot study, undertaken with local government officials in Japan, concerning their involvement in planning for, setting up, and managing evacuation centers for vulnerable populations in Japan during the Great East Japan Earthquake in 2011. The objective is to illuminate the challenges that officials faced, and the resolutions and lessons learned in the preparation of evacuation centers through this event.

Background: Potentially vulnerable population groups in disasters include the elderly and frail, people who are isolated, and those with chronic diseases including mental health conditions or mobility issues. The 2011 Great East Japan Earthquake disaster affected regions of Japan where the proportion of older population is relatively higher than other parts of the country. In 2008, the Japanese Government Cabinet Office implemented guidelines for the preparation and establishment of evacuation centers for vulnerable populations. However, the 2011 disaster highlighted issues regarding the role and responsibility across governments relating to planning, setup, and management of evacuation centers.

Methods: The study was comprised of two phases. The first involved interviews with local government and relevant agencies' officials who have been involved in establishing evacuation centers for vulnerable populations in Japan. Five officials were recruited from the local government area affected by the disaster in Japan. Face-to-face, semi-structured interviews were audio-recorded and thematic analysis was conducted using NVivo software.

Results: Four themes emerged. They were: (1) reflecting on role and responsibility for community, (2) awareness of the need for preparedness, (3) factors causing organizations to be underprepared, and (4) the need for greater community resilience. Conclusion: This pilot study demonstrated that the establishment of clear role descriptions and responsibilities are key for local governments to prepare for the establishment of disaster evacuation centers, particularly for vulnerable populations.

Prehosp Disaster Med 2017;32(Suppl. 1):s125

doi:10.1017/S1049023X17003533
Hospital Surge Capacity in the 2011 Great East Japan

Earthquake and Tsunami

Kazuma Morino

Emergency, Yamagata Prefectural Medical Center for Emergency, Yamagata/Japan

Study/Objective: Until now, there is no experience or evidence about hospital surge capacity in Tsunami disasters in Japan. In the meantime, we had experienced the 2011 Great East Earthquake and Tsunami. So, we will investigate how we make hospital surge capacity in Tsunami disasters.

Background: Surge capacity is a functional expansion capability for catastrophic situations within the organization to deal with a disaster. For hospitals, it can be said that it is the ability of the health care system to accept a large number of patients that occur in a sudden disaster. Not just one of the hospitals, the hospital group, the first-aid station in the area, and more must be considered, as well as the ability of the health care system in the affected prefecture, neighboring prefectures, and nationwide.

Methods: We have investigated five hospitals in Miyagi Prefecture. All hospitals are disaster-based hospitals that were prepared for natural disasters and designated by the local government. We compared bed capacity of these hospitals at peacetime and at the time of disaster; how they effected their surge capacity, and the regional bed capacity. We studied bed capacity in Yamagata Prefecture and places next to Miyagi Prefecture at that time.

Results: Two of the five hospitals that were near the pacific coast should install additional (extra) beds. The number of beds were about two or three times short of daily new admissions. Another two of five that were placed at inland hospitals had no need for additional beds. All hospitals stopped ordinary work to make or expand their capacity of beds and medical staff. Yamagata Prefecture could make slightly more bed capacity.

Conclusion: Except big hospitals in the affected area by Tsunami, hospitals were required to expand their additional (extra) beds for two or three times the daily new admissions, medical staff, and equipment suitable for disaster situations.

Prehosp Disaster Med 2017;32(Suppl. 1):s125

doi:10.1017/S1049023X17003545

Evacuation Burden of a Safety-Net Academic Medical Center during Hurricane Sandy: Implications for

Reverse Triage

Christopher P. Wang ${ }^{1}$, Rushabh Shab ${ }^{2}$, Sidrah Malik', Ian Portelli ${ }^{1}$, Lewis R. Goldfrank ${ }^{1}$, Silas W. Smith ${ }^{1}$

1. Ronald O. Perelman Department Of Emergency Medicine, New York University School of Medicine, New York/NY/United States of America

2. New York University School of Medicine and Stern School of Business, New York/NY/United States of America

Study/Objective: We describe evacuation burdens of a municipal, safety-net academic medical center, following the largest Atlantic hurricane in United States history.

Background: Typically applied to hospitals receiving surge capacity, reverse triage models have suggested that up to 
50-60\% of hospitalized patients are dischargeable in acute disaster. In this case, reverse triage occurred in practice in an evacuating facility to minimize interfacility transfers.

Methods: A retrospective review of the electronic records of patients evacuated from Bellevue Hospital Center during Hurricane Sandy and its aftermath, from October 30 - November 2, 2012 was undertaken. Demographic and clinical data, equipment needs, ambulatory status, transport requirements, forensic status, and ultimate disposition were evaluated.

Results: A total of 732 patients were admitted to the hospital or undergoing treatment in the emergency department at landfall. Of these, 723 records $(98.8 \%)$ were available for review. Only 226 (31.3\%) patients could be discharged home; 38 (5.3\%) were discharged to shelters, while the remaining 459 (63.4\%) patients required transfer to neighboring hospitals, subacute nursing facilities, or correctional facilities for further care. There were $236(32.6 \%)$ either non-ambulatory or demonstrated gait instability; 66 (9.1\%) patients were being treated in intensive care settings, including 16 (2.2\%) patients who were ventilator dependent, and 19 critical neonatal patients. There were $324(44.8 \%)$ patients admitted to inpatient psychiatry. Patients were directly transported to at least 37 individual facilities in multiple hospital networks.

Conclusion: Pragmatically, we found a lower incidence of dischargeable patients than previously assumed. The burden placed on hospital staff, evacuation teams, and neighboring hospitals during evacuation of a large, urban, quaternary care public hospital is severe. Simultaneous citywide evacuation of multiple hospitals may be untenable without prior plans to coordinate resources for such large-scale healthcare system stresses. This study highlights the need to carefully reconsider evacuation, operational and modeling assumptions and solutions in at-risk healthcare infrastructures in cities across the country.

Prehosp Disaster Med 2017;32(Suppl. 1):s125-s126

doi:10.1017/S1049023X17003557

DMAT Operation in 2016 Kumamoto Earthquake Hisayoshi Kondo, Yuichi Koido, Yoshitaka Kohayagawa, Mibo Misaki, Yuzuru Kawashima, Yuji Kondo, Ayako Takahashi Dmat Office, Disaster Medical Center of Japan, Tachikawa, Tokyo/ Japan

Study/Objective: Evaluation of the Japan DMAT activities in a recent domestic major earthquake, referring to learnings from the past.

Background: The Japan DMAT system was established in 2005. At the time of the 2011 Great East Japan Earthquake, 1,852 members responded and provided hospital operation support and patient air evacuation. However, we found that the command system, safety and function screening of clinics and small hospitals via Emergency Medical Information System (EMIS), operation hand over to the subacute disaster phase, and logistic support needs to be improved. We have evaluated if those findings improved in the 2016 Kumamoto Earthquake operation.

Methods: We evaluated all 466 who responded, DMAT post activity reports and investigated any improved activities from the past responses.
Results: There were 2,071 DMAT members who responded. Among 10-day operation, the EMIS system was utilized to screen the level of damages to the clinics, small hospitals and also the evacuation shelters. The DMAT logistic team was activated and resulted in rapid replenishment of medical supplies to the damaged hospitals, and helped shifting of the command system from onset of earthquake to the subacute phase.

Conclusion: Compared to the past, the Kumamoto Earthquake had less trauma patients even though there were a lot of collapsed housing. People stayed inside of their own car due to fear from collapse. This declined activity of daily living in all ages, and created major needs in public health and welfare improvements. It is expected that the Nankai trough Earthquake may result in the biggest damages to Japan. Therefore, with our experiences, we must establish everyday cooperation and drills with local public health services, to operate quick responses to maintain and improve public health. Also, we must establish the psychological first aid system for the patients and the rescuers, which includes DMAT, and needs to cooperate with building inspections personnel to secure the safety of medical support in the damaged buildings.

Prehosp Disaster Med 2017;32(Suppl. 1):s126

doi:10.1017/S1049023X17003569

\section{Mitigating Matthew: 5 Lessons to Help Improve Hurricane Hospital Preparedness}

Lancer A. Scott

Division of Emergency, Medical University of South Carolina, Charleston/SC/United States of America

Study/Objective: Following Hurricane Katrina, US hospitals have largely improved their approach to hurricane preparedness. Yet the timing and uncertainty of hurricanes present unique challenges for hospitals and emergency preparedness officials. Here we present the experience of one coastal hospital directly in the path of Hurricane Matthew (October 2016).

Background: Hurricane Matthew made US landfall on October 8, 2016 near McClellanville, South Carolina, just north of Charleston. The storm caused nearly $\$ 10-15$ billion in damages along the southeast coastline, representing the 22nd most damaging storm in US history.

Methods: This presentation "from the field", documents one coastal hospital's experience preparing for, responding to and recovering from Hurricane Matthew.

Results: Key lessons addressed to better prepare hospitals for hurricanes, include decision making regarding evacuation or shelter in place, evaluation of clinical services to maintain during the storm, the preparation and organization of staff, and the importance of developing an early recovery process to resume hospital operations. Conclusion: In retrospect, the massive mobilization of resources may have been safely modulated downward without risk. But the potential for damage was real and the early call to evacuate was the right call. Our hospital experienced a committed, compassionate and coordinated response; and with minor modifications, coastal hospitals that follow simple rules should be ready.

Prehosp Disaster Med 2017;32(Suppl. 1):s126

doi:10.1017/S1049023X17003570 